\title{
Combinatorial Approaches and Conjectures for 2-Divisibility Problems Concerning Domino Tilings of Polyominoes
}

\author{
Lior Pachter
}

Department of Mathematics

MIT, Cambridge, MA 02139

lpachter@math.mit.edu

Submitted: September 24, 1997; Accepted: November 8, 1997

\begin{abstract}
We give the first complete combinatorial proof of the fact that the number of domino tilings of the $2 n \times 2 n$ square grid is of the form $2^{n}(2 k+1)^{2}$, thus settling a question raised in [4] . The proof lends itself naturally to some interesting generalizations, and leads to a number of new conjectures.

Mathematical Subject Classification. Primary 05C70.
\end{abstract}

\section{Introduction}

The number of domino tilings of the $n \times m$ square grid was first calculated in a seminal paper by Kasteleyn [6]. He showed that, for $n, m$ even, the number of tilings $N(n, m)$ is given by

$$
N(n, m)=\prod_{j=1}^{\frac{n}{2}} \prod_{k=1}^{\frac{m}{2}}\left(4 \cos ^{2} \frac{\pi j}{n+1}+4 \cos ^{2} \frac{\pi k}{m+1}\right) .
$$

This result, while interesting in its own right, does not reveal all of the properties of $N(n, m)$ at first glance. For example, $N(2 n, 2 n)$ is either a perfect square or twice a perfect square (this was first proved by Montroll [7] using linear algebra and later proved by Jokusch [5] and others). Another interesting observation is that

$$
N(2 n, 2 n)=2^{n}(2 k+1)^{2} .
$$


A derivation of this fact from $(\underline{\mathbf{1}})$ has been obtained independently by a number of authors; we refer the reader to [4] . A combinatorial proof of $(\underline{2})$ has proved more elusive, although partial results have been established [2] . As we shall show, a direct combinatorial proof of $(\underline{\mathbf{2}})$ illuminates the combinatorics behind $N(2 n, 2 n)$ and leads directly to generalizations.

Interestingly, perhaps because of the closed form of equation ( $\underline{\mathbf{1}})$, observations other than the ones mentioned above have been scarce. Propp has remarked [9] that "Aztec diamonds and their kin have (so far) been much more fertile ground for exact combinatorics than the seemingly more natural rectangles".

We hope to show that there is a rich source of problems to be found in the enumeration of perfect matchings of rectangular grids. In fact, it seems that the tools needed to resolve many of the problems have yet to be discovered.

\section{The square grid}

Theorem 1 Let $N(2 n, 2 n)$ be the number of domino tilings of the $2 n \times 2 n$ square grid.

$$
N(2 n, 2 n)=2^{n}(2 k+1)^{2} .
$$

Our proof is broken down into two parts. The first part is not new, in fact it appears as a very special case in a theorem in [2]. Since we are interested in this special case only, we provide a simplified version of the proof in [2] that sacrifices much of the generality but illustrates the elegant combinatorial nature of the argument.

We begin by introducing the notation we will use. Rather than discussing perfect matchings of graphs, we will use the dual graph and think of edges in the perfect matching as dominoes covering two adjacent squares. We will, on occasion, use the two descriptions interchangeably. For an arbitrary region $R$, we will use the notation $\# R$ for the number of domino tilings of $R$. For example,

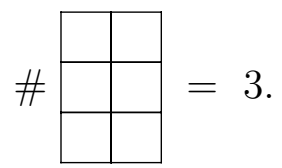

We will use the notation $\#_{2} R$ for the parity of the number of domino tilings of $R$.

The direction of a domino from a fixed square is either up, down, left or right. We shall say that a domino is oriented in the positive (resp. negative) direction from a given square if its direction is up or to the right (resp. down or to the left). For example, in the tiling below, the top left square has a domino that is positively oriented and whose direction is right.

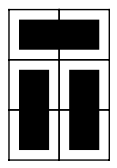

Lemma 1 Label the diagonal squares on the $2 n \times 2 n$ square grid from the bottom left to the top right with the labels $a_{1}, b_{1}, a_{2}, b_{2}, \ldots, a_{n}, b_{n}$. The number of domino 
tilings of the square grid with dominoes placed at $a_{1}, a_{2}, \ldots, a_{n}$ is dependent only on the orientation of the dominoes and not their direction.

Figure 1 illustrates the labeling of the diagonal for the $8 \times 8$ square grid:

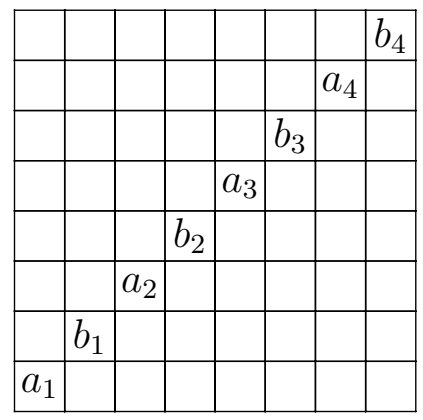

Figure 1

Proof of lemma: Let $M$ be any domino tiling of the $2 n \times 2 n$ square grid. Let $M^{\prime}$ be the tiling obtained by reflecting $M$ across the diagonal and define $D=M \cup M^{\prime}$ ( $D$ is allowed to consist of multiple dominoes). Notice that in the dual graph of the $2 n \times 2 n$ square grid, $D$ is a 2 -factor and is therefore a disjoint union of even-length cycles. Furthermore, since $D$ is symmetric across across the diagonal, any cycle maps to another cycle under the reflection.

Now define $C_{i}^{\prime}$ to be the cycle containing $a_{i} . C_{i}^{\prime}$ can have at most one other vertex on the diagonal because every vertex in $C_{i}^{\prime}$ has degree 2 . Furthermore, such a vertex must be of the type $b_{j}$, for otherwise the number of vertices enclosed by $C$ is odd (contradicting the fact that $D$ is a disjoint union of even length cycles). It follows that all the cycles $C_{i}^{\prime}$ are distinct.

Finally, let $C_{i}=C_{i}^{\prime} \cap M$ be the alternating cycles (cycles in the dual graph alternating between edges in the tiling and edges not in the tiling) in $M$ obtained from $C_{i}^{\prime}$. By the above arguments, the alternating cycles $C_{i}$ are disjoint. Thus, there is a bijection between any two sets of tilings with fixed dominoes of the same orientation on the $a_{i}$ 's. We simply select all the dominoes on the $a_{i}$ 's that have switched direction and rotate the appropriate alternating cycles.

Example 1 Changing the direction of the domino at $a_{2}$ we have

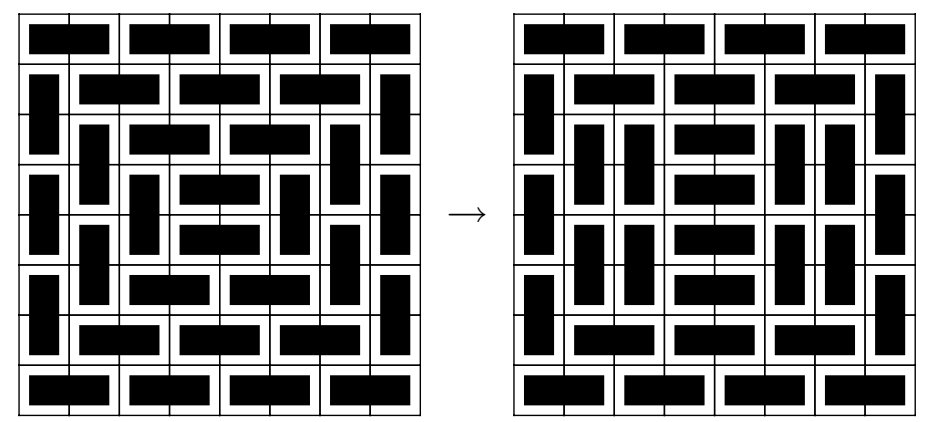


We now define a class of grids, $H_{n}$ (first introduced by Ciucu [2] ), as follows:
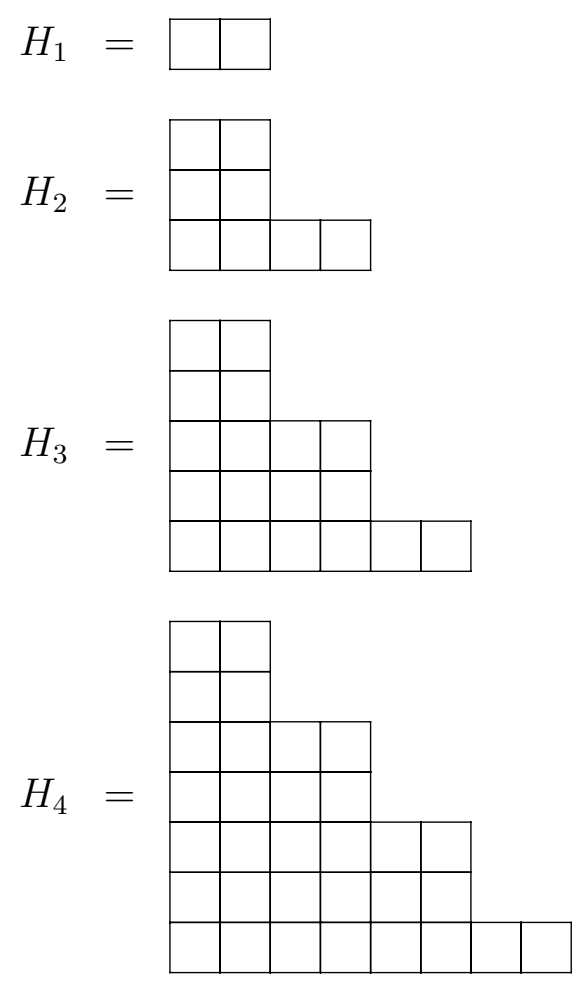

$\vdots \quad \vdots$

$H_{n}$ is defined from $H_{n-1}$ by adding a grid of size $2 \times(2 n-1)$ to the left of $H_{n-1}$.

Lemma 2 The number of domino tilings of the square grid is given by

$$
N(2 n, 2 n)=2^{n}\left(\# H_{n}\right)^{2} \text {. }
$$

Proof of lemma: Consider a fixed orientation for the dominoes covering the $a_{i}$ 's. We can assume (using Lemma 1) that the directions of the dominoes are all either down or to the right (call such a configuration reduced). Notice that the square grid decomposes naturally into two halves. Figure 2 illustrates an example of a reduced configuration.

\begin{tabular}{|l|l|l|l|l|l|l|l|}
\hline$U$ & $U$ & $U$ & $U$ & $U$ & $U$ & $U$ & $U$ \\
\hline$U$ & $U$ & $U$ & $U$ & $U$ & $U$ & & \\
\hline$U$ & $U$ & $U$ & $U$ & $U$ & $U$ & & \\
\hline$U$ & $U$ & $U$ & $U$ & & & & \\
\hline$U$ & $U$ & $U$ & $U$ & & & & \\
\hline$U$ & $U$ & & & & & & \\
\hline$U$ & $U$ & & & & & & \\
\hline & & & & & & & \\
\hline
\end{tabular}

Figure 2 
Notice that the region filled with $U$ is equivalent to $H_{n}$, as is its complement. Now consider the standard checkerboard 2-coloring of the square grid. All the U's which are adjacent to empty squares have the same color. It follows that in any reduced configuration, every domino covers either two $U$ 's or none at all. We have from Lemma 1 that

$$
N(2 n, 2 n)=2^{n} \sum_{C} \# C
$$

where $C$ ranges over all reduced configurations. From the remarks above it follows that

$$
\sum_{C} \# C=\left(\# H_{n}\right)^{2}
$$

which completes the proof of the lemma.

Lemma $3 \# H_{n}$ is odd.

Proof of lemma: Our proof is by induction. The case when $n=1,2$ is trivial. We illustrate the general case by showing the step $n=3 \Rightarrow n=4$.

Begin by observing that
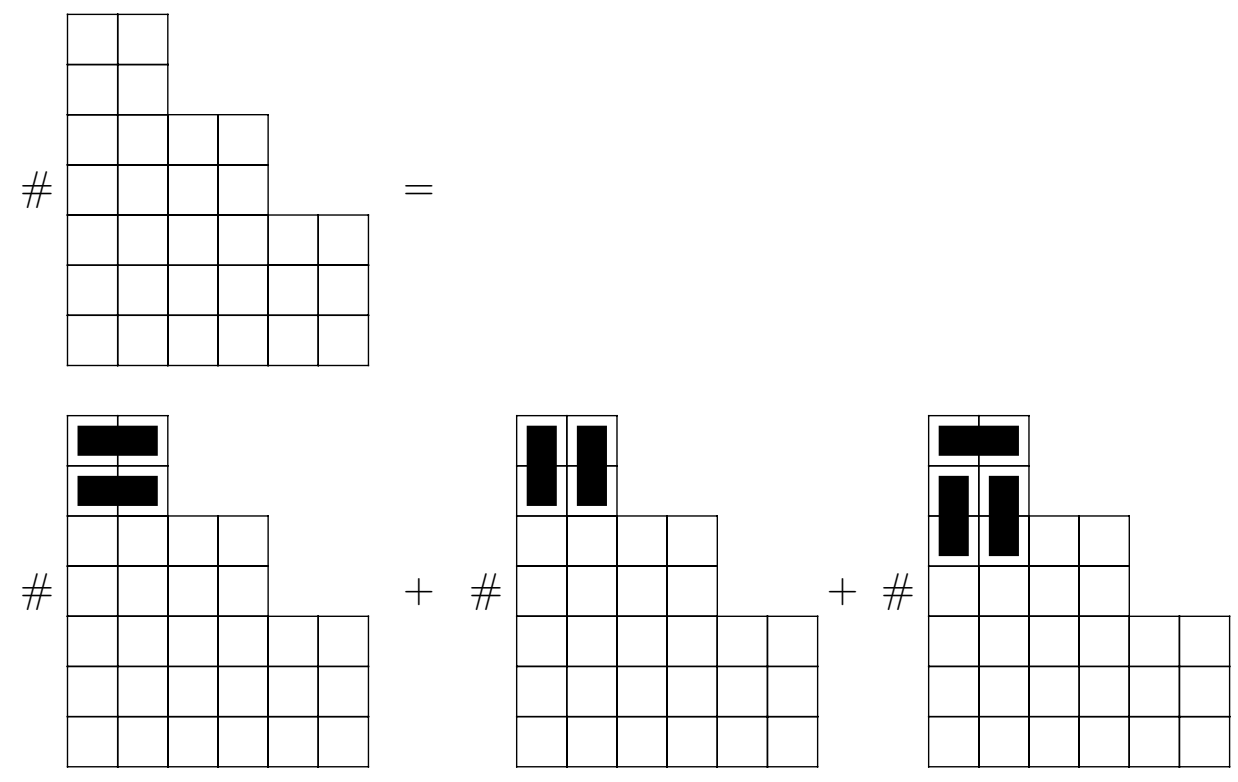

The first two terms in $(\underline{7})$ are equal, so we have

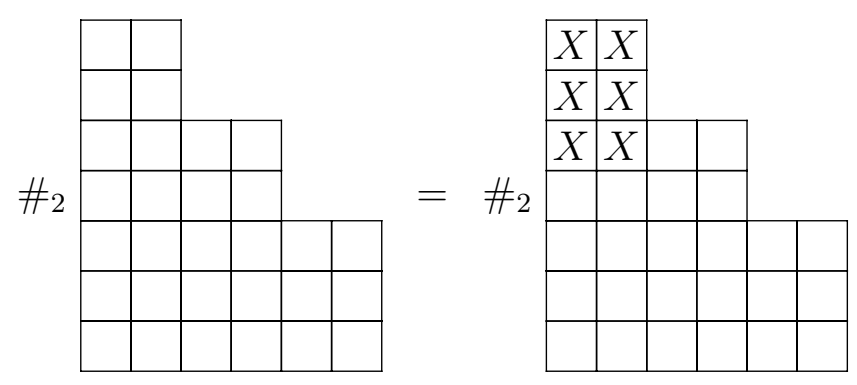


where the $X$ 's denote squares that cannot be used.

We now begin removing shapes of the form \begin{tabular}{r|r|}
$X$ & $X$ \\
\hline$X$ \\
\hline$X$
\end{tabular} from the diagonal, using a similar idea:
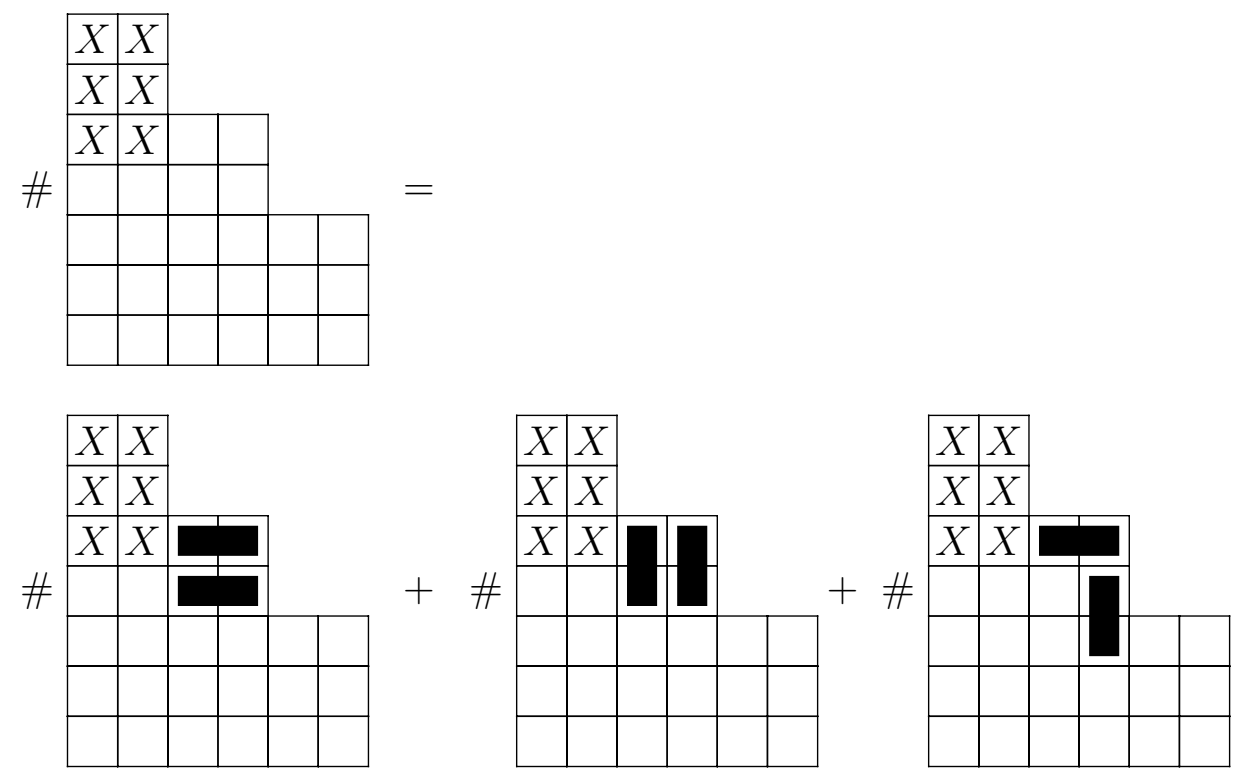

Hence, we can conclude that
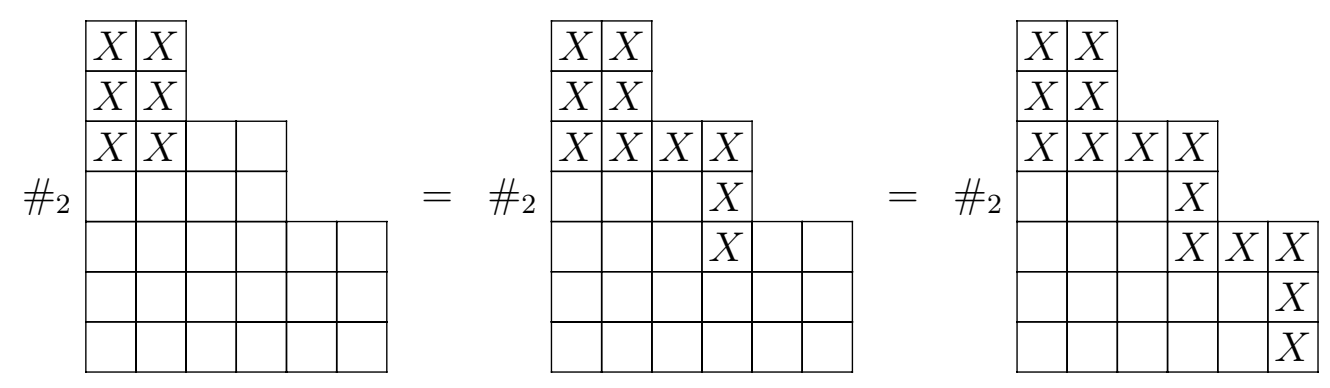

Our last shape is $H_{n-1}$ (minus the forced domino on the bottom right), flipped and rotated by $90^{\circ}$ ! It follows that

$$
\#_{2} H_{n}=\#_{2} H_{n-1}
$$

Proof of theorem: The theorem follows immediately by applying Lemmas 2 and 3.

\section{Rectangular Grids}

The exact formula for the largest power of 2 appearing in $N(2 n, 2 n)$ suggests an investigation of the same question for $n \times m$ rectangular grids.

We use the notation $(a, b)$ to denote the greatest common divisor of $a$ and $b$. 
Problem 1 Let $N(n, m)$ be the number of domino tilings of the $n \times m$ rectangular grid. Prove combinatorially that

$$
\begin{aligned}
N(2 n, 2 m) & =2^{\frac{(2 n+1,2 m+1)-1}{2}}\left(2 r_{1}+1\right) \\
N(2 n+1,2 m) & =2^{\frac{(n+1,2 m+1)-1}{2}(3+j)}\left(2 r_{2}+1\right)
\end{aligned}
$$

where $j$ is defined by $n+1=2^{j}(2 t+1)$. (In the above $r_{1}, r_{2}, t$ are natural numbers that may vary for different $n, m$.)

Equation (12) [4] . (This has been observed by Saldanha [10] ). Indeed, the other case should follow by similar methods. A combinatorial proof is not known for either case. Combinatorial proofs are important in this context because other methods fail for regions that are more complicated. Section 4 contains numerous examples where an analogous formula to ( $\underline{\mathbf{1}})$ is lacking, and therefore there is no closed form formula from which to work.

Stanley [11] has conjectured that for fixed $m$ (and $n$ varying), $N(n, m)$ satisfies a linear recurrence with constant coefficients that is of order $2^{\frac{m+1}{2}}$ (he established this when $m+1$ is an odd prime). Such recurrences have been obtained for small $m$ and can be used to provide proofs of special cases of Problem $\underline{1}$. Indeed, Bao [1] has used such recurrences together with the reduction techniques we use above to establish combinatorial proofs for the formulas in Problem 1 for $n \leq 2$. Unfortunately, the difficulty in establishing recurrences for $N(n, m)$ combinatorially probably precludes the general applicability of the above method for finding combinatorial proofs for (12) and (13).

Equation (13), which remains to be verified using algebraic methods, was checked extensively for various values of $n$ with $m \leq 10$.

\section{Conjectures}

\subsection{Deleting From Diagonals}

We begin with an intriguing "power of 2" conjecture for a new type of region we call the spider.

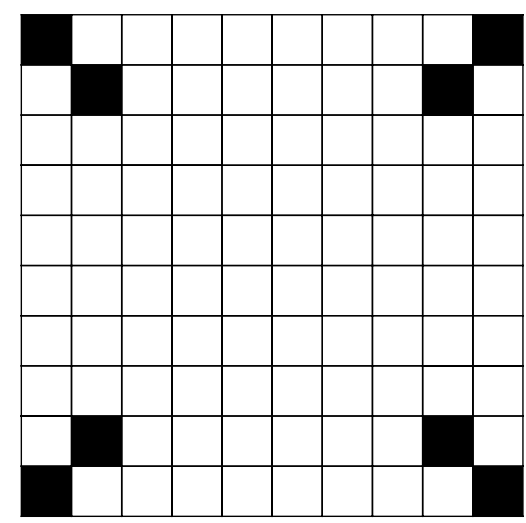

The $(5,2)$ spider 
Define the $(n, k)$ spider to be the region obtained by deleting $k$ consecutive squares (from the corner) along each diagonal of the $2 n \times 2 n$ square grid.

Conjecture 1 Let $S(n, k)$ be the number of domino tilings of the $(n, k)$ spider.

$$
S(n, k)=2^{n+k(2 k-1)}(2 r+1), \quad k \leq\left\lfloor\frac{n}{2}\right\rfloor .
$$

When $k>\left\lfloor\frac{n}{2}\right\rfloor$ the region reduces to an Aztec diamond after the removal of forced dominoes (for a definition and extensive discussion of Aztec diamonds see [3] ). If $n$ is even we see that $(\underline{\mathbf{1 4}})$ reduces to the formula for the number of domino tilings of the Aztec diamond when $k=\frac{n}{2}$. Conjecture 1 has been checked numerically for $n \leq 10$.

Values of $S(n, k)$ for $n=\{2, \ldots, 6\}, k \leq\left\lfloor\frac{n}{2}\right\rfloor$

\begin{tabular}{|l|l|l|l|l|l|}
\hline$n / k$ & 2 & 3 & 4 & 5 & 6 \\
\hline 0 & $2^{2} 3^{2}$ & $2^{3} 29^{2}$ & $2^{4} 17^{2} 53^{2}$ & $2^{5} 241^{2} 373^{2}$ & $2^{6} 5^{4} 31^{2} 53^{2} 701^{2}$ \\
\hline 1 & $2^{3}$ & $2^{4} 7^{2}$ & $2^{5} 13^{4}$ & $2^{6} 3^{4} 11^{2} 139^{2}$ & $2^{7} 5^{2} 744397^{2}$ \\
\hline 2 & - & - & $2^{10}$ & $2^{11} 31^{2}$ & $2^{12} 3617^{2}$ \\
\hline $3-$ & - & - & - & - & $2^{21}$ \\
\hline
\end{tabular}

\subsection{Deleting From Step Diagonals}

The acute reader will have noticed that the arguments in Lemma 1 establish that any domino tiling of the $2 n \times 2 n$ square grid contains at least $n$ disjoint alternating cycles. The tiling in Example 1 illustrates that this is the best result possible (for other results along these lines see [8] ). Figure 3 shows how to place $n$ dominoes so as to ensure the remaining figure has only one tiling (the $n$ dominoes "block" the $n$ cycles).

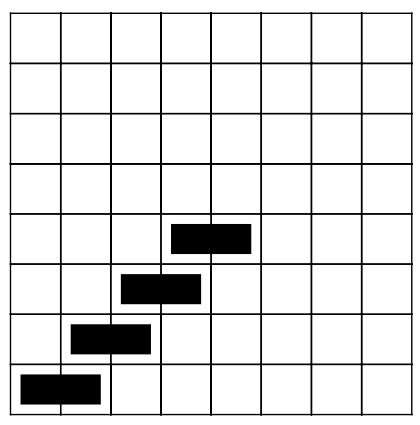

Figure 3

We shall call the set of the first $n$ stepwise horizontal edges in the $2 n \times 2 n$ square grid the step-diagonal.

The above observation has led Propp [9] to ask whether removal of only half the dominoes from the bottom of the step diagonal results in a graph whose number of tilings is interesting. Indeed, drawing on his idea, we have formulated the following remarkable conjecture: 
Conjecture 2 Let $G$ be the grid obtained after the removal of any $k$ edges from the step-diagonal of the $2 n \times 2 n$ square grid. Then the number of domino tilings of $G$ is of the form

$$
\# G=2^{n-k}(2 r+1)
$$

In addition, if the $k$ edges removed are consecutive from the lower left corner then $2 r+1$ is a perfect square.

Also related to the step-diagonal is the following conjecture:

Conjecture 3 Let $G$ be the grid obtained after the removal of one edge from the stepdiagonal of the $2 n \times 2 n$ square grid. Using the notation that $N(2 n, 2 n)=2^{n}(2 k+1)^{2}$, the number of domino tilings of $G$ satisfies:

$$
\# G=c(2 k+1)
$$

where $c$ is a constant which depends upon which edge was removed.

Conjecture 2 was checked extensively for $n \leq 10$ (the exponential growth of the number of configurations to be tested precluded exhaustive checking of this conjecture). Conjecture 3 was checked for all $n \leq 10$.

Edward Early has considered the number of tilings of holey squares. The holey square $H(n, m)$ is a $2 n \times 2 n$ square with a hole of size $2 m \times 2 m$ removed from the center. He has conjectured

\section{Conjecture 4}

$$
\# H(n, m)=2^{n-m}(2 k+1)^{2} .
$$

The fact that $2^{n-m} \mid H(n, m)$ is easily obtained using Lemma 1 (the fact that $H(n, m)$ is either a perfect square or twice a perfect square also follows). The fact that $n-m$ is the highest power of 2 dividing $H(n, m)$ does not follow inductively in this case. Bao [1] has established that the conjecture is true for $m=1,2$ by showing that a region similar to $H_{n}$ has an odd number of domino tilings. Unfortunately, algebraic methods using ( $\underline{\mathbf{1}})$ fail in this case since no analogous formulas from which to work are known.

Finally, based on numerical evidence, we present our grand conjecture:

Conjecture 5 Conjecture 2 is true for all holey squares (with $n$ replaced by $n-m$ in (15)). Conjecture 3 is true for all holey squares (with $(2 k+1)$ replaced by the square

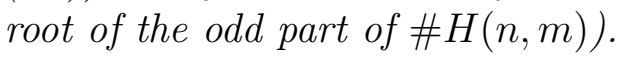

\section{Conclusion}

The results and conjectures of the previous sections point to an underlying combinatorial principle which is most likely the basis of the nice patterns of powers of 2 . While such a result eludes us, the following old (somewhat forgotten) result which appears in [7] may hint at an algebraic approach to "power of 2" conjectures:

Proposition 1 A graph $G$ has an even number of perfect matchings iff there is a non-empty set $S \subseteq V(G)$ such that every point is adjacent to an even number of points of $S$. 


\section{Acknowledgments}

We thank Joshua Bao and Jim Propp for helpful suggestions and comments. Special thanks go to Glenn Tesler for helping to draw the tiling pictures and to David Wilson for providing his program vax.el with which all the conjectures were tested. Finally, we are indebted to the anonymous referee for excellent suggestions which greatly helped in improving the final version of the paper.

\section{References}

[1] J. Bao, On the number of domino tilings of the rectangular grid, the holey square, and related problems, Final Report, Research Summer Institute at MIT (1997).

[2] M. Ciucu, Enumeration of perfect matchings in graphs with reflective symmetry, J. Combin. Theory Set. A 77 (1997), no. 1, p 67-97.

[3] N. Elkies, G. Kuperberg, M. Larsen and J. Propp, Alternating-Sign Matrices and Domino Tilings, Journal of Algebraic Combinatorics 1 (1994) p 111-132 and p 219-234.

[4] P. John, H. Sachs and H. Zernitz, Problem 5. Domino Covers in Square Chessboards, Zastosowania Matematyki (Applicationes Mathematicae) XIX, 3-4 (1987), p 635-641.

[5] W. Jokusch, Perfect matchings and perfect squares, J. Comb. Theory Ser. A 67 (1994), p 100-115.

[6] P. W. Kasteleyn, The statistics of dimers on a lattice, I: The number of dimer arrangements on a quadratic lattice, Physica 27 (1961), p 1209-1225.

[7] L. Lovász, Combinatorial Problems and Exercises, North-Holland Publishing Company (1979).

[8] L. Pachter and P. Kim, Forcing Matchings on Square Grids, preprint (1996).

[9] J. Propp, Twenty Open Problems in Enumeration of Matchings, preprint (1996).

[10] N. Saldanha, personal communication.

[11] R. Stanley, On dimer coverings of rectangles of fixed width, Discrete Applied Mathematics, 12 (1985), no. 1, p 81-87. 